\title{
Different Effects of Oral Contraceptive and Dydrogesterone Treatment on Oxidative Stress Levels in Premenopausal Women
}

\author{
Jui-Tung Chen ${ }^{\text {a, c }}$, Kazuhiko Kotani ${ }^{\text {b }}$
}

\begin{abstract}
Background: The aim of the study was to observe the changes in blood oxidative stress levels by oral contraceptive (OC) and/or dydrogesterone (DG) treatment.

Methods: A retrospective cohort of 27 premenopausal women with primary dysmenorrhea consisted of the OC treatment group $(\mathrm{N}=17)$ and the DG treatment group $(\mathrm{N}=10)$ by choice of the initial treatment. The OC group included two subgroups: patients with continuous $\mathrm{OC}$ treatment (treated for at least 15 months, $\mathrm{N}$ $=10$ ) and patients with discontinuous OC treatment (switched to DG treatment after approximately 6 months of initial OC treatment: $\mathrm{N}=7$ ). The DG group had 15 months of continuous DG treatment. Blood parameters, including diacron-reactive oxygen metabolites (d-ROMs: an oxidative stress marker), were measured.
\end{abstract}

Results: The d-ROMs level was elevated in the OC group 3 months after initial treatment (mean: from 321 (at baseline) to 512 Carratelli Units (Carr U); P $<0.01$ ), while such changes were not observed in the DG group. The d-ROMs level was reduced in the discontinuous OC subgroup 15 months after initial treatment (from 508 (3 months after initial treatment) to 372 Carr U; P < 0.01 ), while such changes were not observed in the continuous OC subgroup. The DG group displayed unchanged the d-ROMs level.

Conclusion: Replacing OC with DG can attenuate oxidative stress as elevated by $\mathrm{OC}$, thereby alleviating the possible vascular risks with OC treatment.

Keywords: d-ROMs test; Dydrogesterone; Ethinyl estradiol; Norethisterone; Oral contraceptives; Oxidative stress

Manuscript submitted December 8, 2017, accepted December 18, 2017

aJui-Tung Chen Clinic, Tokyo, Japan

bepartment of Community and Family Medicine, Jichi Medical University, Tochigi, Japan

${ }^{c}$ Corresponding Author: Jui-Tung Chen, J.T. Chen Clinic, 2-13-5 Akasaka, Akasaka-Kaikan B1F, Minato-Ku, Tokyo 107-0052, Japan.

Email: chin-cl@jcom.zaq.ne.jp

doi: https://doi.org/10.14740/jocmr3307w

\section{Introduction}

Dysmenorrhea is a common health problem among young women [1]. The monthly disability results in 600 million lost working hours and a productivity loss of two billion dollars annually in the United States [1]. A better understanding of the treatment of dysmenorrhea is therefore beneficial for both the affected individuals and their society.

Oral contraceptives (OCs) [2] and progestin [3] have been reported to be effective for the treatment of dysmenorrhea. Some studies have pointed out the risk of thromboembolism associated with the use of hormonal contraceptives [4], while the results concerning the risk have been conflicting across studies [5]. As women who take OC for dysmenorrhea use it for the long term, vascular complications among young women must be seriously considered [6]. In contrast, oral administration of dydrogesterone (DG), the 6-dehydro reversal isomeride of progesterone with molecular structure extremely similar to natural progesterone, at a daily dose between 5 and $60 \mathrm{mg}$, shows beneficial vascular effects [3] and may have advantages over other progestogen-combined hormonal replacement treatment administered to avoid estrogen-induced adverse effects [7]. Cyclic application of DG has also been shown to induce regular menstruation with symptomatic relief, reduced blood loss, and fewer days of bleeding in women with dysmenorrhea [3].

Biologically, DG treatment reduces reactive oxygen species (ROS) formation [8] and exerts modulating effects on nitric oxide (NO) synthesis and on the expression of endothelial NO synthase (eNOS) [9]. This can be one of the mechanisms of the several beneficial effects of DG. Although the adverse effects of OCs have not yet been elucidated, overproduction of ROS (leading to an increase in oxidative stress) by OCs can be associated with vascular dysfunction [10]. We have previously reported that there were cross-sectionally higher blood levels of diacron reactive oxygen metabolites (d-ROMs: an oxidative stress marker) in women undergoing OC treatment [11], leading to a hypothesis that $\mathrm{OC}$ treatment can elevate oxidative stress levels.

While the examination of ROS is sometimes difficult in the clinic setting, a test for d-ROMs has been developed [12]. This test reflects the concentration of hydroperoxides [12] and has been established as a clinically applicable oxidative stress marker [13]. The present study aimed at investigating blood 
Table 1. Clinical Background of Study Subjects

\begin{tabular}{|c|c|c|c|}
\hline Parameter & OC group $(\mathrm{N}=17)$ & DG group $(\mathrm{N}=10)$ & $\mathbf{P}$ \\
\hline Age, years & $37.5 \pm 9.8$ & $41.4 \pm 6.1$ & 0.27 \\
\hline Current smokers, n (\%) & $2(12 \%)$ & $1(10 \%)$ & 0.99 \\
\hline Systolic blood pressure, $\mathrm{mm} \mathrm{Hg}$ & $115 \pm 18$ & $109 \pm 10$ & 0.31 \\
\hline Diastolic blood pressure, $\mathrm{mm} \mathrm{Hg}$ & $73 \pm 13$ & $70 \pm 9$ & 0.55 \\
\hline Platelet, $\times 10^{4} / \mu \mathrm{L}$ & $26.1 \pm 5.0$ & $29.0 \pm 6.6$ & 0.20 \\
\hline Hemoglobin A1c, \% & $4.8 \pm 0.2$ & $4.9 \pm 0.3$ & 0.31 \\
\hline LDL-cholesterol, mg/dL & $100 \pm 31$ & $98 \pm 26$ & 0.87 \\
\hline HDL-cholesterol, mg/dL & $75 \pm 20$ & $73 \pm 10$ & 0.75 \\
\hline d-ROMs, Carr U & $321 \pm 61$ & $356 \pm 70$ & 0.19 \\
\hline $\mathrm{BAP}, \mu \mathrm{M}$ & $2,033 \pm 179$ & $1,953 \pm 191$ & 0.28 \\
\hline
\end{tabular}

OC: oral contraceptive; DG: dydrogesterone; WBC: white blood count; LDL: low-density lipoprotein; HDL: high-density lipoprotein; ROMs: reactive oxygen metabolites; BAP: biological antioxidant potential. Data are presented as means \pm standard deviations, medians (interquartile ranges) or subject numbers (\%). P < 0.05 (significance level): comparison between the OC and DG groups by an unpaired $t$-test or Fisher's exact test.

oxidative stress levels, as assessed by the d-ROMs test, in premenopausal women receiving OC and/or DG treatment.

\section{Materials and Methods}

\section{Study subjects}

This study was a retrospective cohort design based on our clinic's database (between April 2010 and November 2013). The ethics committee of our institution approved this study and informed consent was obtained from subjects. For avoiding a selection bias, the subjects were chosen from all cases included in the original database. Inclusion criteria were non-pregnant and premenopausal subjects, those who were free from coronary artery disease, cerebrovascular disease, diabetes mellitus, and liver or renal disease, as well as those who were taking any medications, including antioxidant supplements. As the first study cohort, the subjects who complained of primary dysmenorrhea and received OC or DG treatment according to their condition $[2,3]$ during at least a 15 -month period were chosen $(\mathrm{N}=40)$. The subjects, who had received OC or DG treatment during the period, included some cases, in whom OC treatment was switched to DG treatment after approximately 6 months of initial OC treatment for the following reasons: headache $(\mathrm{N}=1)$, skin redness $(\mathrm{N}=2)$, and financial issues $(\mathrm{N}=4)$, respectively. As the final study cohort, exclusion criteria were the subjects who had not received blood tests (i.e., parameters related to oxidative stress and inflammation) on at least three-time points (baseline (pre-treatment), 3 months af- ter initial treatment, and approximately 15 months after initial treatment) (excluded subjects: $\mathrm{N}=13$ ).

The study cohort ultimately consisted of 27 women (initial OC treatment group: $\mathrm{N}=17$; initial $\mathrm{DG}$ treatment group: $\mathrm{N}$ $=10$ ). Among the initial OC treatment group, two subgroups existed as follows: patients with continuous OC treatment $(\mathrm{N}=$ 10) and patients with discontinuous OC treatment (as switched from initial $\mathrm{OC}$ treatment to $\mathrm{DG}$ treatment: $\mathrm{N}=7$ ). The data were compared in the initial OC and DG treatment groups between baseline and 3 months after initial treatment (phase 1). Subsequent data were further compared in the subgroups with continuous and discontinuous OC treatment, besides the initial DG treatment group, between 3 months after initial treatment and 15 months after initial treatment (phase 2).

Estimating a sample size for the present study was based on a previous study that examined the difference in the dROMs level, a primary outcome, between OC users (mean: 380 Carratelli Unit (Carr U)) and non-users (325 Carr U) [11]. With the power (1-beta level) of $80 \%$ at the alpha level of $5 \%$ to detect a difference of 55 Carr $U$ of the d-ROMs levels and a standard deviation of 40 Carr $U$, a sample size of 8 in a group was estimated.

\section{Drugs}

In this study, the OC treatment was a triphasic preparation consisting of 21 tablets of $0.035 \mathrm{mg}$ of ethinyl estradiol (EE) and 12 tablets of $0.5 \mathrm{mg}$ of norethisterone (NET) or nine tablets of $1.0 \mathrm{mg}$ of NET. The subjects received the combination of EE and NET for 21 days during each 28-day interval. For the DG 
Table 2. Changes in Data From Baseline to 3 Months After Initial Treatment

\begin{tabular}{|c|c|c|c|}
\hline Parameter & Baseline & 3 months & $\mathbf{P}$ \\
\hline d-ROMs, Carr U & & & $<0.01 * *$ \\
\hline OC group $(\mathrm{N}=17)$ & $321 \pm 61$ & $512 \pm 79$ & \\
\hline $\mathrm{BAP}, \mu \mathrm{M}$ & & & 0.21 \\
\hline OC group $(\mathrm{N}=17)$ & $2,033 \pm 179$ & $2,029 \pm 161$ & \\
\hline High-sensitivity CRP, mg/dL & & & $0.01 *$ \\
\hline OC group $(\mathrm{N}=17)$ & $0.02(0.01-0.05)$ & $0.10(0.03-0.22)$ & \\
\hline DG group $(\mathrm{N}=10)$ & $0.01(0.01-0.02)$ & $0.01(0.01-0.02)$ & \\
\hline $\mathrm{WBC}, / \mu \mathrm{L}$ & & & 0.28 \\
\hline OC group $(\mathrm{N}=17)$ & $5,688 \pm 1,319$ & $5,994 \pm 1,134$ & \\
\hline DG group $(\mathrm{N}=10)$ & $29.0 \pm 6.6$ & $29.4 \pm 5.5$ & \\
\hline Fibrinogen, $\mathrm{mg} / \mathrm{dL}$ & & & 0.06 \\
\hline OC group $(\mathrm{N}=17)$ & $275 \pm 78$ & $278 \pm 68$ & \\
\hline DG group $(\mathrm{N}=10)$ & $237 \pm 27$ & $234 \pm 28$ & \\
\hline LDL-cholesterol, mg/dL & & & 0.75 \\
\hline OC group $(\mathrm{N}=17)$ & $100 \pm 31$ & $108 \pm 31$ & \\
\hline DG group $(\mathrm{N}=10)$ & $98 \pm 26$ & $103 \pm 24$ & \\
\hline
\end{tabular}

OC: oral contraceptive; DG: dydrogesterone; WBC: white blood coun; LDL: low-density lipoprotein; HDL: high-density lipoprotein; ROMs: reactive oxygen metabolites; BAP: biological antioxidant potential. Data are presented as means \pm standard deviations or medians (interquartile ranges). ${ }^{*}$ $<0.05,{ }^{* *} \mathrm{P}<0.01$ : comparison between the OC and DG groups by two-way repeated measure ANOVA.

treatment, the subjects took DG orally $10 \mathrm{mg} /$ day.

\section{Measurements}

All the subjects were interviewed for their medical history, menstruation information and current smoking status. Measurements of body mass index, blood pressure and blood parameters during fasting were taken. Blood was sampled within 2 weeks after menstruation or withdrawal bleeding. As blood parameters, hemoglobin, white blood cell count (WBC), platelet count, hemoglobin A1c (HbAlc), low-density lipoprotein cholesterol (LDL-C), triglycerides, high-density lipoprotein cholesterol (HDL-C), fibrinogen, and high-sensitivity C-reactive protein (hs-CRP) were measured with standard methods in a single laboratory center that is nationally certified (Mitsubishi BCL Laboratory Co. Ltd, Tokyo, Japan). Concerning specific blood parameters of oxidative stress, the d-ROMs test was accomplished by the Free Radical Analytical System (Diacron, Grosseto, Italy) according to the analytical manual [12]. The d-ROMs test unit of measurement is the Carr $U$ and the test shows intra- and inter-assay coefficients of variations of $<$ $3 \%$ [13]. The biological antioxidant potential (BAP) level was determined simultaneously by the system; this test assesses the ability to reduce ferric ions to ferrous ions as an anti-oxidative reaction [14]. The level is expressed as $\mu \mathrm{M}$ [14].

\section{Statistical analysis}

Comparison of each parameter between the groups was performed with an unpaired $t$-test or Fisher's exact test, and oneway analysis of variance (ANOVA) or two-way (time $\times$ group) repeated measure ANOVA. Comparison of pre- and post-data of each parameter within each group was performed with a paired $t$-test. The triglycerides and hs-CRP values were logtransformed in all analyses, due to their skewed distributions. A P value of $<0.05$ was considered significant.

\section{Results}

Clinical background of the patients in each study cohort group is shown in Table 1. There were no obvious differences in the clinical background between the groups with initial OC treatment and initial DG treatment.

Table 2 shows the data at baseline and 3 months after initial treatment (phase 1). The d-ROMs level became elevated in the 
Table 3. Changes of Data From 3 to 15 Months After Initial Treatment

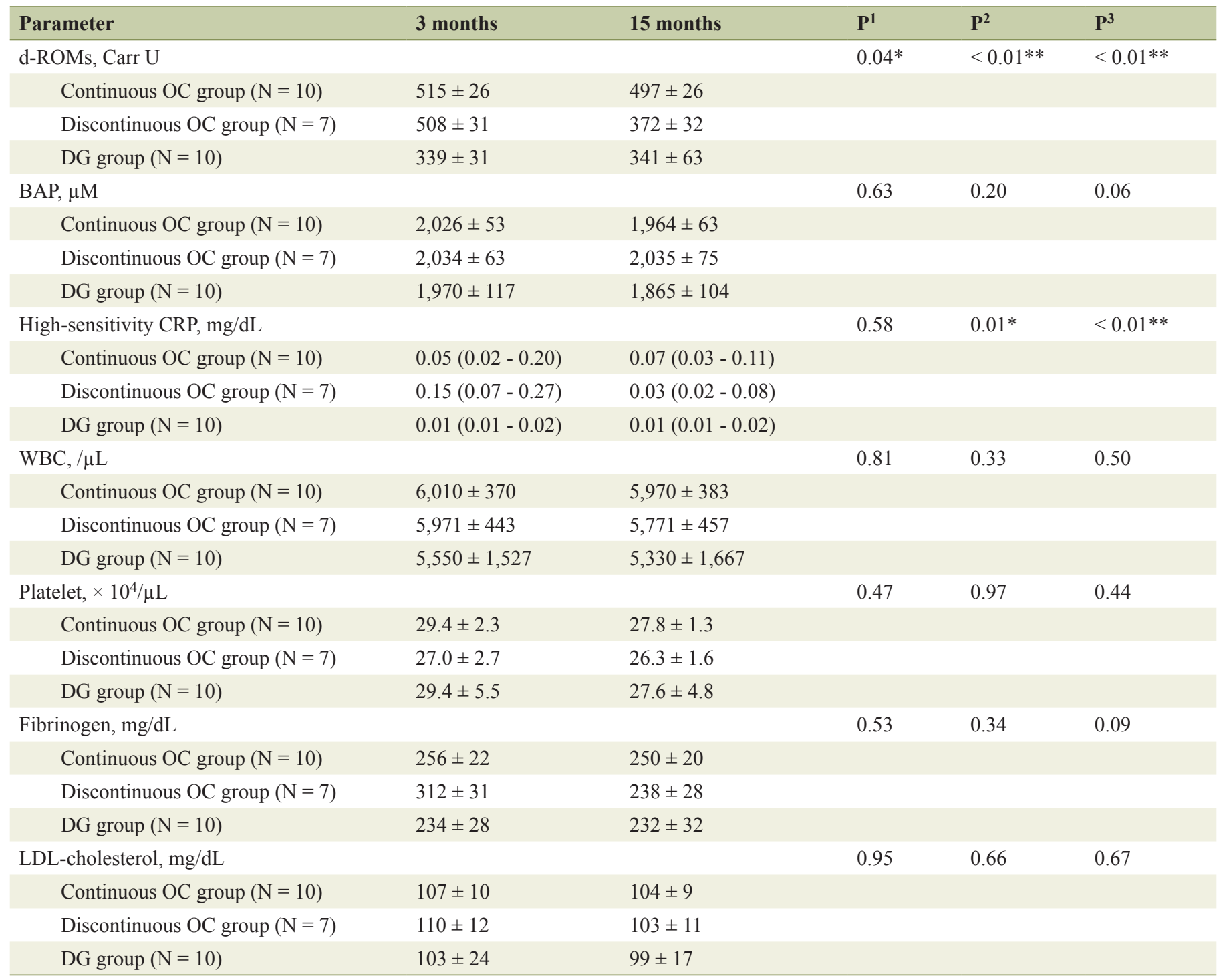

OC: oral contraceptive; DG: dydrogesterone; WBC: white blood count; LDL: low-density lipoprotein; HDL: high-density lipoprotein; ROMs: reactive oxygen metabolites; BAP: biological antioxidant potential. Data are presented as means \pm standard deviations or medians (interquartile ranges). * $P$ $<0.05$, ${ }^{*} \mathrm{P}<0.01$ : comparison by two-way repeated measure ANOVA. $\mathrm{P}^{1}$ : between the continuous and discontinuous OC groups; $\mathrm{P}^{2}$ : between the continuous $O C$ and DG groups; $P^{3}$ : between the discontinuous OC and DG groups.

initial OC treatment group (mean: from 321 to 512 Carr U; P < 0.01 ), while the d-ROMs level was largely unchanged in the initial DG treatment group (from 356 to 339 Carr U; $\mathrm{P}=0.51$ ). The change in the d-ROMs level was significantly different between the two groups. The hs-CRP level also became elevated in the initial OC treatment group (median: from 0.02 to $0.10 \mathrm{mg} / \mathrm{dL}$; $\mathrm{P}<0.01$ ), while the hs-CRP level was largely unchanged in the initial DG treatment group (from 0.01 to $0.01 \mathrm{mg} / \mathrm{dL} ; \mathrm{P}=0.93$ ). The change in the hs-CRP level was significantly different between the two groups. The changes in the other parameters, such as BAP, WBC, platelets, fibrinogen and LDL-C, did not show significant differences between the two groups.

Table 3 shows the data at 3 months after initial treatment and at approximately 15 months after initial treatment (phase
2). After approximately 6 months of initial OC treatment, the subgroup with discontinuous OC treatment was switched to DG treatment. The data at 3 months after initial treatment did not significantly differ between the groups with continuous OC treatment, discontinuous OC treatment and DG treatment. Between the subgroups with continuous OC treatment and discontinuous OC treatment, the change in the d-ROMs level was significantly different: namely, the d-ROMs level was reduced in the subgroup with discontinuous OC treatment (mean: from 508 to 372 Carr U; P $<0.01$ ), while the d-ROMs level was largely unchanged in the subgroup with continuous OC treatment (from 515 to 497 Carr $\mathrm{U} ; \mathrm{P}=0.70$ ). The reduced d-ROMs level in the subgroup with discontinuous OC treatment (mean: 372 Carr U) seemed to be mostly near the baseline d-ROMs 


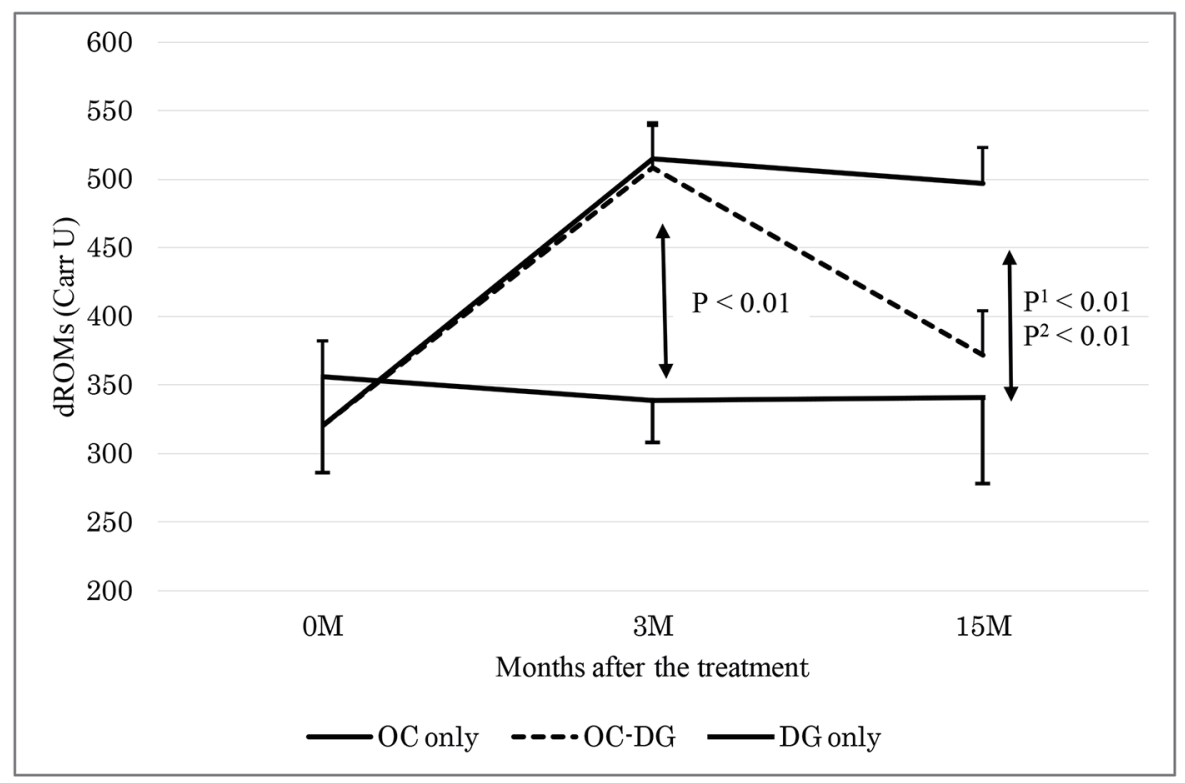

Figure 1. Changes of $d-R O M s 3$ and 15 months after the treatment. OC: oral contraceptive; DG: dydrogesterone; OC-DG: initial OC treatment was switched to DG treatment. Data are presented as means \pm standard deviations. P: OC ( $3 \mathrm{M})$ versus DG only groups 0 and 3 months after the treatment; $P^{1}$ : OC versus DG; $P^{2}$ : OC-DG versus DG only groups 3 and 15 months after the treatment by two-way repeated measure ANOVA.

level in the OC treatment group (mean: 321 Carr U). The changes in the other parameters, such as BAP, hs-CRP, WBC, platelets, fibrinogen and LDL-C, did not show significant differences between the two subgroups.

As shown in Table 3, the DG treatment group displayed unchanged levels of d-ROMs, BAP, hs-CRP, WBC, platelets, fibrinogen and LDL-C. At 3 and 15 months after initial treatment, the changes in the d-ROMs level and the hs-CRP level were significantly different between the DG treatment group and the subgroup with continuous OC treatment, and between the DG treatment group and the subgroup with discontinuous OC treatment (Figs. 1 and 2). The changes in the other param-

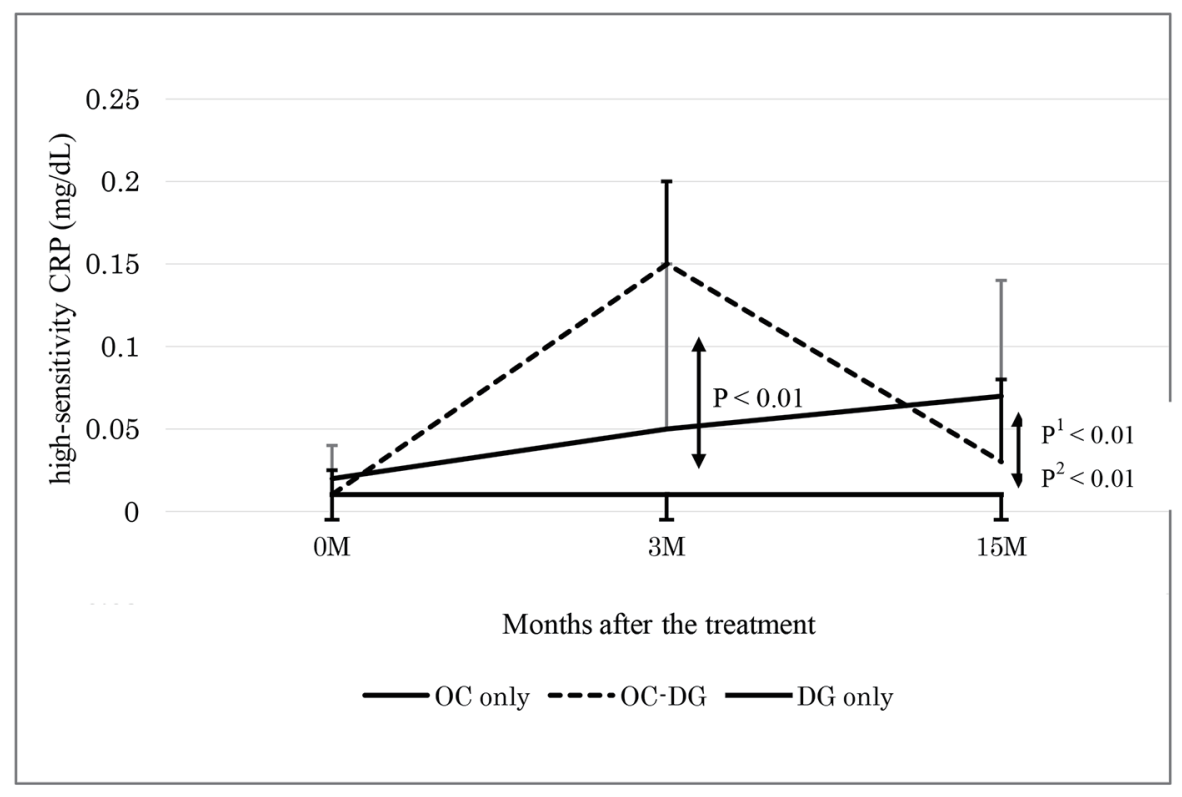

Figure 2. Changes of high-sensitivity CRP 3 and 15 months after the treatment. OC: oral contraceptive, DG: dydrogesterone, OC-DG: initial OC treatment was switched to DG treatment. Data are presented as medians (interquartile ranges). P: OC (3 M) versus DG groups 0 and 3 months after the treatment; $P^{1}$ : OC versus DG; $P^{2}$ : OC-DG versus DG only groups 3 and 15 months after the treatment by two-way repeated measure ANOVA. 
eters, such as BAP, WBC, platelets, fibrinogen and LDL-C, did not show significant differences between the DG treatment group and the continuous or discontinuous OC treatment subgroups.

There were three current smokers (one in the continuous OC treatment subgroup, one in the discontinuous OC treatment subgroup, and one in the DG treatment group). Their smoking habits did not change during the study period (from baseline to 15 months after initial treatment), even though smoking can generally be a factor potentially associated with oxidative stress and inflammation.

\section{Discussion}

This study demonstrated that OC treatment increased blood oxidative stress levels in premenopausal women, as measured by the d-ROMs test, a primary outcome measure. Following a previous cross-sectional study that showed a high d-ROMs level in women undergoing OC treatment [11], it would be valuable to demonstrate the finding with $\mathrm{OC}$ treatment in a follow-up study. On the other hand, DG treatment did not produce any changes in the oxidative stress level. Notably, when OC treatment was changed to DG treatment, the increased oxidative stress level produced by the OC treatment returned to the baseline level in at least 6 months. This may suggest a possible risk reduction in vascular complications caused by use of OCs. Therefore, it may be important for premenopausal women to switch from OC treatment to DG treatment temporarily to reduce the oxidative burden.

The mechanisms responsible for the increase in oxidative stress by OCs remain unclear, while there are several points to be considered for possible explanations and to discuss further perspectives. The behavior of molecules related to oxidative stress can differ according to the types and doses of estrogen, progestogen, or the particular compounds of estrogen and progestogen. Estrogens are generally known to have various vascular actions by increasing the bioavailability of $\mathrm{NO}$ via activation of NO synthase, and NO is inversely related to oxidative stress. However, some differences between estradiol (E2) and EE have been demonstrated, and EE (as used in our current study) seemingly cannot alleviate oxidative stress [15]. NO production and the viability of endothelial cells after exposure to hydrogen peroxide was found to be increased by E2 but not by EE [15], NO production in aortic cells from ovariectomized rats was increased by $\mathrm{E} 2$ but reduced with $\mathrm{EE}$ [16], and a microarray gene test indicated that the NO synthase-coded gene was down-regulated by OC treatment [14]. Moreover, a clinical study reported that OCs increased the blood levels of oxidative stress markers (e.g., lipid peroxides) and decreased the levels of antioxidant markers (e.g., gammatocopherol and beta-carotene) in women [17]. Thus, OC treatment, especially that containing $\mathrm{EE}$, can increase oxidative stress levels.

Concerning treatment with progesterone, its beneficial effects have been shown in the form of improvement of atherogenic lipid profiles [8] and ROS formation (and in turn, oxidative stress production) [10]. However, there is a contra- diction in that progesterone can enhance nicotinamide adenine dinucleotide phosphate (NADPH) oxidase activity and ROS formation [18]. An animal study has demonstrated that conjugated equine estrogen suppresses the development of atherosclerosis, but not in combination with medroxyprogesterone acetate [19]. Medroxyprogesterone acetate could interfere with the vasoprotective effects of estrogens, while this was not observed to happen with natural endogenous progesterone [19]. These results suggest that all progestogens are not equal in terms of their hormonal profiles and actions, for instance, in the abilities of the progestogens to bind to progesterone receptors or to other steroid receptors [20]. One opinion is that progesterone, DG or 17-hydroxyprogesterone caproate are drugs that do not produce some of the adverse effects associated with other progestogens [20], and DG exerts endothelial anti-inflammatory actions (i.e., via a decrease in expression of leukocyte adhesion molecules) [20], perhaps linking with modulation of oxidative stress. Clinical studies have also found that DG treatment could improve oxidative stress conditions in combination with E2 [21]. The DG component offers endometrial protection and cycle control without negating the vasoprotective effects of E2 [22]. Thus, DG treatment may not increase oxidative stress; rather, it may counteract an increase in oxidative stress levels caused by OC treatment [23].

It will be necessary to discuss the types and doses of progestogen in combination with EE in OC treatment, while a progestogen-alone oral preparation is reported to have no adverse effects with respect to vascular complications [24]. Indeed, the MEGA study [25] found that the risk of venous thrombosis was positively associated with estrogen dose and by the type of progestin in combination. However, this finding was not supported by the Danish historical cohort study in that only small differences in the risks of thrombotic stroke and myocardial infarction were observed according to the type of progestin [6]. Progestogen can antagonize the beneficial effects of estrogen by modulating estrogen-induced activation of protein $\mathrm{C}$ resistance [26]. Again, progestogen-only drugs were reported to confer no increased risk of venous thromboembolism, when taken in the form of low-dose norethisterone pills, as desogestrel-alone agents, or in the form of hormone-releasing intrauterine devices [24]. In a general sense, progestin-alone treatment is thought to pose little risk of thromboembolism and is recommended for women at high risk of thromboembolism and young women with primary dysmenorrhea who need long-term treatment [27]. Although in OC treatment (including NET) of the present study, the NET-alone effects on oxidative stress levels were not completely evaluated, we think little effects of NET on oxidative stress, even though the effects existed from the above evidence.

To ameliorate this side effect of OCs, the uses of vitamins $\mathrm{E}$ and C [28], or products containing green tea catechins [29] were reported to control the oxidative stress generated by OCs. However, we are convinced that temporary replacement of OC with DG treatment is an infallible method for reducing the burden of oxidative stress in women with primary dysmenorrhea.

A follow-up study style was the strength of this study. This study had the limitations. The study design was retrospective, and the study cohort was chosen from subjects in whom all the pertinent test results were available (the tests were not planned 
beforehand). The sample size was relatively small. Only one measurement method was used to evaluate the blood oxidative stress level. A prospective, larger study or blinded randomized trials with additional oxidative stress measurements is necessary to establish the results of this study. At present, we do not have much evidence of the adverse effects of OCs on vascular dysfunction in association with oxidative stress [10], and future studies may include vascular outcomes. The precise different effects of types and doses of OCs may also be a future debate. Anyhow, more accumulation of data of oxidative stress in DG treatment is necessary for patient care and public health implication.

In conclusion, the present study showed that OC treatment in premenopausal women elevated the oxidative stress level, while DG treatment did not elevate the level. When OC treatment was replaced by DG treatment, the oxidative stress level was reduced. These findings suggest that temporary replacement of OC with DG treatment can attenuate the elevated oxidative stress by $\mathrm{OC}$, thereby alleviating the possible risks of vascular complications induced by OC treatment. Further research is warranted to confirm these findings.

\section{Conflict of Interest}

The authors report no conflict of interest.

\section{Financial Support}

None.

\section{References}

1. Coco AS. Primary dysmenorrhea. Am Fam Physician. 1999;60(2):489-496.

2. Winkler UH, Ferguson H, Mulders JA. Cycle control, quality of life and acne with two low-dose oral contraceptives containing 20 microg ethinylestradiol. Contraception. 2004;69(6):469-476.

3. Aydar CK, Coleman BD. Treatment of primary dysmenorrhea: a double-blind study. JAMA. 1965;192:10031005.

4. Parkin L, Skegg DC, Wilson M, Herbison GP, Paul C. Oral contraceptives and fatal pulmonary embolism. Lancet. 2000;355(9221):2133-2134.

5. Food and Drug Administration Office of Surveillance and Epidemiology. Combined hormonal contraceptives (CHCs) and the risk of cardiovascular disease endpoints (http://www.fda.gov/downloads/Drugs /DrugSafety/ UCM277384.pdf).

6. Lidegaard O, Lokkegaard E, Jensen A, Skovlund CW, Keiding N. Thrombotic stroke and myocardial infarction with hormonal contraception. N Engl J Med. 2012;366(24):2257-2266.

7. Gutierrez J, Ballinger SW, Darley-Usmar VM, Landar A. Free radicals, mitochondria, and oxidized lipids: the emerging role in signal transduction in vascular cells. Circ Res. 2006;99(9):924-932.

8. Schneider C, Jick SS, Meier CR. Risk of cardiovascular outcomes in users of estradiol/dydrogesterone or other HRT preparations. Climacteric. 2009;12(5):445-453.

9. Ross RL, Serock MR, Khalil RA. Experimental benefits of sex hormones on vascular function and the outcome of hormone therapy in cardiovascular disease. Curr Cardiol Rev. 2008;4(4):309-322.

10. Simoncini T, Caruso A, Giretti MS, Scorticati C, Fu XD, Garibaldi S, Baldacci C, et al. Effects of dydrogesterone and of its stable metabolite, 20-alpha-dihydrodydrogesterone, on nitric oxide synthesis in human endothelial cells. Fertil Steril. 2006;86(4 Suppl):1235-1242.

11. Chen JT, Kotani K. Oral contraceptive therapy increases oxidative stress in pre-menopausal women. Int J Prev Med. 2012;3(12):893-896.

12. Trotti R, Carratelli M, Barbieri M, Micieli G, Bosone D, Rondanelli M, Bo P. Oxidative stress and a thrombophilic condition in alcoholics without severe liver disease. Haematologica. 2001;86(1):85-91.

13. Verde V, Fogliano V, Ritieni A, Maiani G, Morisco F, Caporaso N. Use of N,N-dimethyl-p-phenylenediamine to evaluate the oxidative status of human plasma. Free Radic Res. 2002;36(8):869-873.

14. De Groote D, Perrier d'Hauterive S, Pintiaux A, Balteau B, Gerday C, Claesen J, Foidart JM. Effects of oral contraception with ethinylestradiol and drospirenone on oxidative stress in women 18-35 years old. Contraception. 2009;80(2):187-193.

15. Andozia MB, Vieira CS, Franceschini SA, Torqueti Tolloi MR, Silva de Sa MF, Ferriani RA. Ethinylestradiol and estradiol have different effects on oxidative stress and nitric oxide synthesis in human endothelial cell cultures. Fertil Steril. 2010;94(5):1578-1582.

16. Bolego C, Cignarella A, Ruzza R, Zaarour C, Messi E, Zanisi M, Puglisi L. Differential effects of low- and highdose estrogen treatments on vascular responses in female rats. Life Sci. 1997;60(25):2291-2302.

17. Pincemail J, Vanbelle S, Gaspard U, Collette G, Haleng J, Cheramy-Bien JP, Charlier C, et al. Effect of different contraceptive methods on the oxidative stress status in women aged 4048 years from the ELAN study in the province of Liege, Belgium. Hum Reprod. 2007;22(8):2335-2343.

18. Wassmann K, Wassmann S, Nickenig G. Progesterone antagonizes the vasoprotective effect of estrogen on antioxidant enzyme expression and function. Circ Res. 2005;97(10):1046-1054.

19. Adams MR, Register TC, Golden DL, Wagner JD, Williams JK. Medroxyprogesterone acetate antagonizes inhibitory effects of conjugated equine estrogens on coronary artery atherosclerosis. Arterioscler Thromb Vasc Biol. 1997;17(1):217-221.

20. Schindler AE. Progestational effects of dydrogesterone in vitro, in vivo and on the human endometrium. Maturitas. 2009;65(Suppl 1):S3-11.

21. Kuba VM, Teixeira MA, Meirelles RM, Assumpcao CR, Costa OS. Dydrogesterone does not reverse the cardiovascular benefits of percutaneous estradiol. Climacteric. 
2013;16(1):54-61.

22. Foster RH, Balfour JA. Estradiol and dydrogesterone. A review of their combined use as hormone replacement therapy in postmenopausal women. Drugs Aging. 1997;11(4):309-332.

23. Stevenson JC, Panay N, Pexman-Fieth C. Oral estradiol and dydrogesterone combination therapy in postmenopausal women: review of efficacy and safety. Maturitas. 2013;76(1):10-21.

24. Mantha S, Karp R, Raghavan V, Terrin N, Bauer KA, Zwicker JI. Assessing the risk of venous thromboembolic events in women taking progestin-only contraception: a meta-analysis. BMJ. 2012;345:e4944.

25. van Hylckama Vlieg A, Helmerhorst FM, Vandenbroucke JP, Doggen CJ, Rosendaal FR. The venous thrombotic risk of oral contraceptives, effects of oestrogen dose and progestogen type: results of the MEGA case-control study. BMJ. 2009;339:b2921.
26. Kemmeren JM, Algra A, Meijers JC, Tans G, Bouma BN, Curvers J, Rosing J, et al. Effect of second- and thirdgeneration oral contraceptives on the protein $\mathrm{C}$ system in the absence or presence of the factor VLeiden mutation: a randomized trial. Blood. 2004;103(3):927-933.

27. Centers for Disease C, Prevention. U S. Medical Eligibility Criteria for Contraceptive Use, 2010. MMWR Recomm Rep. 2010;59(RR-4):1-86.

28. Zal F, Mostafavi-Pour Z, Amini F, Heidari A. Effect of vitamin $\mathrm{E}$ and $\mathrm{C}$ supplements on lipid peroxidation and GSH-dependent antioxidant enzyme status in the blood of women consuming oral contraceptives. Contraception. 2012;86(1):62-66.

29. Finco A, Belcaro G, Cesarone MR. Assessment of the activity of an oral contraceptive on the levels of oxidative stress and changes in oxidative stress after co-treatment with two different types of physiological modulators with antioxidant action. Contraception. 2011;84(4):418-422. 